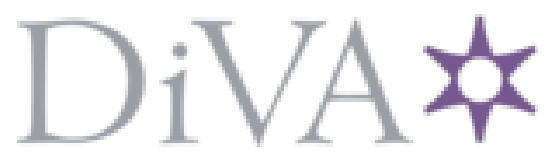

http://www.diva-portal.org

This is the published version of a paper presented at ASME Design Engineering Technical Conferences.

Citation for the original published paper:

Drugge, L., Larsson, T., Berghuvud, A., Stensson, A. (1999)

The nonlinear behaviour of a pantograph current collector suspension.

In: Proceedings of the 1999 ASME Design Engineering Technical Conferences Las Vegas, USA.

N.B. When citing this work, cite the original published paper.

Permanent link to this version:

http://urn.kb.se/resolve?urn=urn:nbn:se:bth-11317 
DETC99/VIB-8026

\title{
THE NONLINEAR BEHAVIOUR OF A PANTOGRAPH CURRENT COLLECTOR SUSPENSION
}

\author{
L. Drugge, T. Larsson, A. Berghuvud and A. Stensson \\ Division of Computer Aided Design \\ Department of Mechanical Engineering \\ Luleå University of Technology \\ SE-971 87 Luleå, Sweden \\ Email: Lars.Drugge@cad.luth.se
}

Keywords: pantograph, current collection, piecewise linear, nonlinear dynamics

\begin{abstract}
The pantograph-catenary system is a critical component for trains required to run at higher speeds. The pantograph often includes nonlinear characteristics and the scope of this work is to investigate if nonlinear dynamic phenomena can occur in an existing design. A model of a pantograph suspension subsystem has been developed according to physical parameter values of the head suspension of the Schunk WBL88/X2 pantograph, providing electric power to the Swedish high-speed train X2 . Studies of the system response for different force excitation show both harmonic, subharmonic and chaotic behaviour for the investigated parameter regions.
\end{abstract}

\section{INTRODUCTION}

A pantograph current collector transfers electric power from an overhead catenary system to a train, see Fig. 1. The pantograph mechanism often consists of a head assembly and a variable-height frame assembly. The frame assembly raises the head assembly into forced contact with the catenary system. The catenary consists of an arrangement of wires suspended from support poles. The catenary is staggered from side to side in a horizontal plane to distribute the wear on the contact head of the pantograph.

As railway companies strive for higher speeds, the problem of satisfactory current collection becomes increasingly important. The performance of the current collection system depends on the dynamic properties of the interacting pantograph and catenary. If the dynamic performance of the system is improved, the variation in contact force is decreased, leading to a reduction in loss of contact, arcing and wear and consequently, possibilities of higher speed.

Pantographs often include geometric nonlinearities, nonlinear stiffness and damping (Poetsch et al., 1997). This indicates that nonlinear phenomena such as subharmonic motion, chaotic motion and multiple solutions might occur (Aidanpää et al., 1994, Natsiavas, 1990, Natsiavas and Gonzales, 1992, Shaw and Holmes, 1983). Models of pantographs which include some nonlinearities have been used in for example ERRI (1992), Larsson and Drugge (1998) and Levy et al. (1968) without special attention to nonlinear phenomena. To investigate if such phenomena can occur in this type of design, a pantograph used on the Swedish high-speed train X2 is studied.

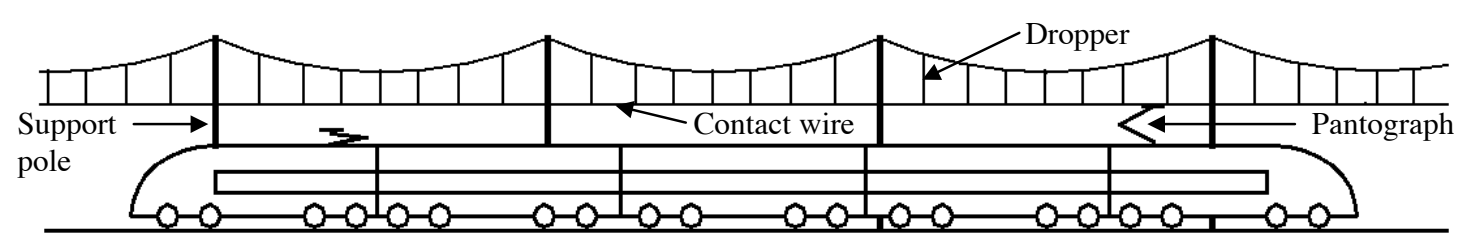

Figure 1. A train with its overhead current collection system. 
In this work, the dynamic behaviour is investigated using numerical simulations. A subsystem of a head assembly suspension is modelled and the system response is studied for different frequencies of harmonic excitation and bias force.

\section{STUDIED SYSTEM}

The pantograph design studied is the Schunk WBL88/X2 pantograph from Schunkgruppen Nordiska AB, Lenhovda, Sweden. It consists of a head assembly and a frame assembly, see Fig. 2. The head assembly includes two carbon collector strips and a head frame. A suspension at each end of the strips, see Fig. 3, allows the carbon collector strips to move individually.

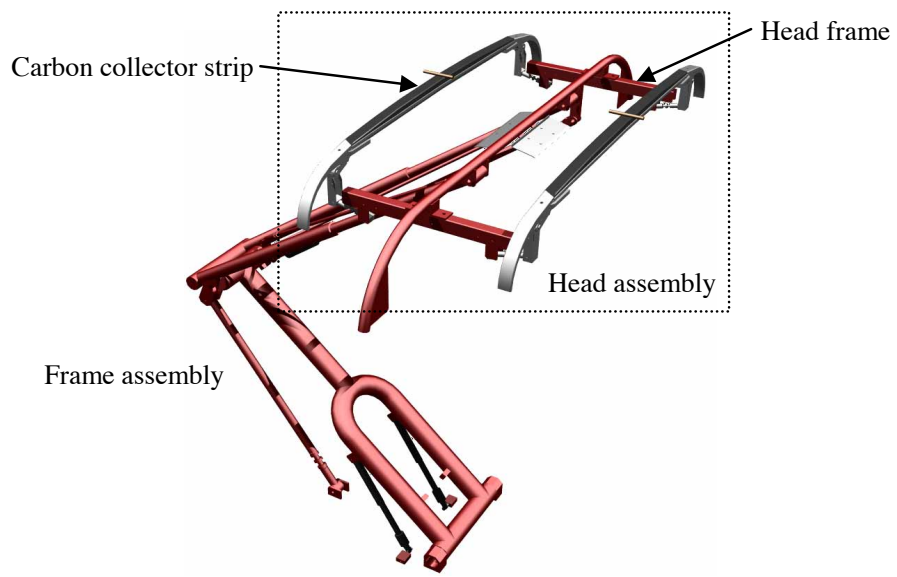

Figure 2. CAD model of a Schunk WBL88/X2 pantograph (Larsson and Drugge, 1998).

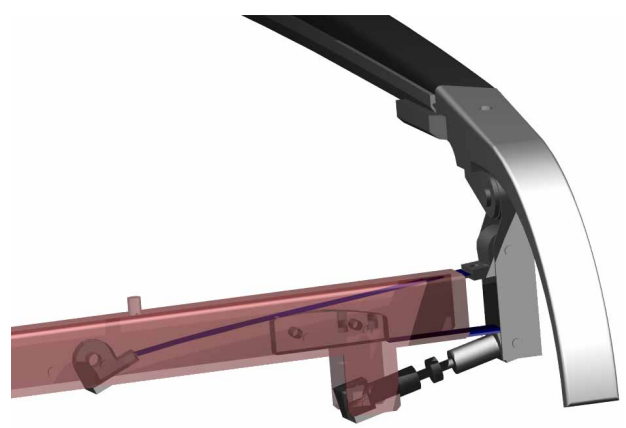

Figure 3. Carbon collector strip suspension.

The suspension, i.e. the studied subsystem, consists of leaf springs and rigid links, see Fig. 4. The two links and the upper leaf spring comprise a four bar linkage which is controlling the vertical movement of the carbon collector strip. The upward displacement of the strip is limited by the head frame and the downward displacement by the lower leaf spring. In the figure the subsystem is shown in its unloaded position, that is, the system is at rest at the upper limit.

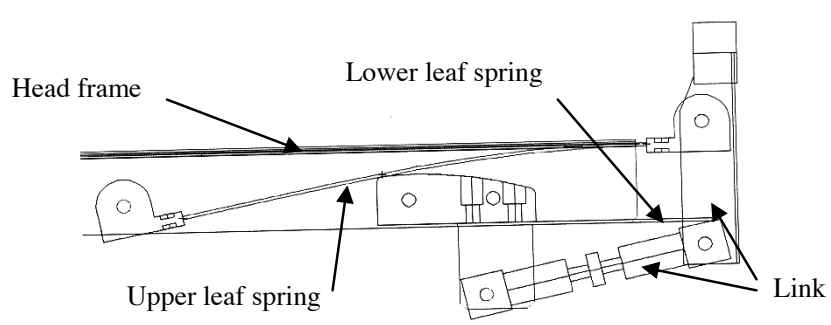

Figure 4. Definition of subsystem parts.

\section{MODELLING}

The main part of the pantograph motion is assumed to occur in the subsystems. Consequently, the head frame is considered to be fixed. Each carbon collector strip is assumed to be excited at mid-point, that is, the zigzag of the catenary is neglected. Due to symmetry, only one subsystem needs to be considered. The dynamics of the leaf springs is assumed to be dominated by the first bending mode. Therefore, the subsystem can be considered as one-dimensional for small amplitudes. The subsystem is modelled as a one degree of freedom system, see Fig. 5, where the characteristics of the suspension is modelled by piecewise linear springs. The system is statically pre-loaded and excited by a harmonic force. The equation of motion for this system is shown in Eq. (1). In Fig. 5 the model is shown in a statically pre-loaded condition.

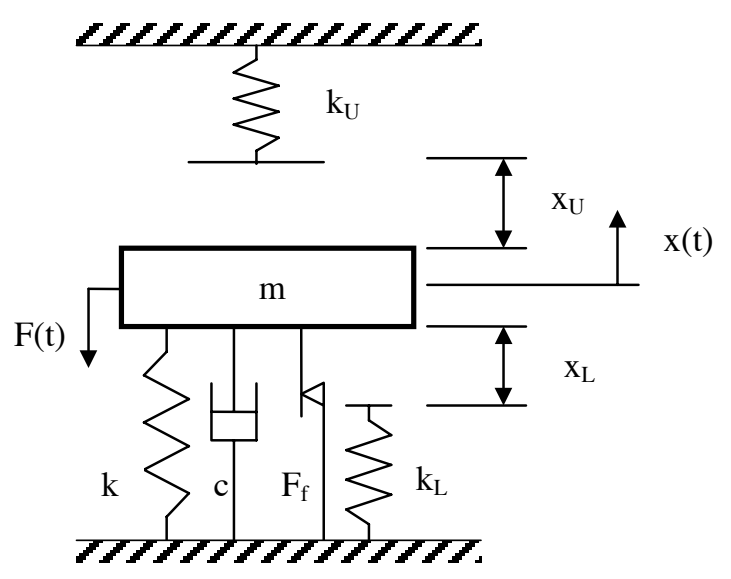

Figure 5. Schematic model of the subsystem.

$$
\begin{gathered}
m \ddot{x}+c \dot{x}+k x+F_{f} \operatorname{sign}(\dot{x})=F(t)+g(x) \\
g(x)=\left\{\begin{array}{cc}
-k_{U}\left(x-x_{U}\right) & x>x_{U} \\
0 & -x_{L} \leq x \leq x_{U} \\
-k_{L}\left(x+x_{L}\right) & x<-x_{L}
\end{array}\right.
\end{gathered}
$$


Table 1. Subsystem parameter values.

\begin{tabular}{cccccc}
\hline $\mathrm{m}[\mathrm{kg}]$ & $\mathrm{k}[\mathrm{N} / \mathrm{m}]$ & $\mathrm{c}[\mathrm{Ns} / \mathrm{m}]$ & $\mathrm{F}_{\mathrm{f}}[\mathrm{N}]$ & $\mathrm{k}_{\mathrm{U}}[\mathrm{N} / \mathrm{m}]$ & $\mathrm{k}_{\mathrm{L}}[\mathrm{N} / \mathrm{m}]$ \\
\hline 1.66 & $1 \cdot 10^{3}$ & 2.6 & 0.15 & $6 \cdot 10^{4}$ & $6.55 \cdot 10^{3}$ \\
\hline
\end{tabular}

The parameter values describing the subsystem are determined from measurements on a full-scale pantograph, at Schunkgruppen Nordiska AB. The damping is assumed to behave as a combination of viscous and Coulomb damping (Larsson and Drugge, 1998). The parameter values are listed in Table 1.

The contact force between the pantograph and the catenary excites the system. The force is mainly composed by a static component and a dynamic component dependent on the running speed and the vibrational behaviour of system (Poetsch et al., 1997). The excitation has a periodicity due to the distance between support poles and droppers (Galeotti et al., 1993). It is assumed that the contact force can be approximated with a static pre-load and a harmonic variation, see Eq. (2). As an example, a train running at a constant speed of $160 \mathrm{~km} / \mathrm{h}$ on a catenary with a pole distance of $60 \mathrm{~m}$ and a dropper distance of $8.8 \mathrm{~m}$ will be excited with a pole frequency of $0.7 \mathrm{~Hz}$ and a dropper frequency of $5 \mathrm{~Hz}$. The pre-load, $\mathrm{F}_{0}$, depends on a static pneumatic uplift force and a velocity dependent aerodynamic uplift force.

$$
F(t)=F_{0}-F_{A} \sin (\omega t)
$$

\section{RESULTS}

The simulations have been performed by numerical integration of the equation of motion described in Eq. 1. Two cases have been considered, see Table 2 .

Table 2. Definition of studied excitations.

\begin{tabular}{ccc}
\hline Case & $\mathrm{F}_{0}[\mathrm{~N}]$ & $\mathrm{F}_{\mathrm{A}}[\mathrm{N}]$ \\
\hline 1 & 12.5 & 6.25 \\
2 & 20.0 & 6.25 \\
\hline
\end{tabular}

In the studied cases, the static pre-load between the fullscale pantograph and catenary is assumed to be $50 \mathrm{~N}$. Thus, the static pre-load of a subsystem is $12.5 \mathrm{~N}$. In the first case, the aerodynamic force contribution is considered to be negligible, such as for a train running at low speed. The second case illustrates a situation when the train is running at higher speed, with a resulting aerodynamic uplift force as consequence.

In Case 1, a pre-load of $12.5 \mathrm{~N}$ corresponds to the displacements $\mathrm{x}_{\mathrm{U}}=0.0125 \mathrm{~m}$ and $\mathrm{x}_{\mathrm{L}}=0.0210 \mathrm{~m}$. For this case, the mass is positioned closer to the upper stop. For Case 2 the corresponding displacements are $\mathrm{x}_{\mathrm{U}}=0.0200 \mathrm{~m}$ and $\mathrm{x}_{\mathrm{L}}=0.0135$ $\mathrm{m}$, which results in a system that is vibrating closer to the lower stop.

Some examples of responses for Case 1 are shown in Fig. 6 as time histories and phase plane portraits. In the phase plane it is easier to identify a periodicity in the response since a closed trajectory will be found for any repetitive phenomenon. To obtain these diagrams, a set of initial conditions are chosen and the equation of motion is solved. The transient part of the motion is neglected and the characteristics of the motion during 100 periods of excitation are plotted. The first three seconds of the time histories are shown. A dash-dotted line represents the periodicity of the excitation force. Examples of response for Case 2 are shown in Fig. 7

To investigate the behaviour of the system for different excitation frequencies, some characteristic measures of the motion are used. The mapping used are Poincare sections and velocities at impact. Poincaré sections represent a trajectory in the phase plane sampled once during each period of excitation. If the response is periodic with the same period as the excitation, the points will converge after a transient to one single point in the phase plane. If the response is periodic with a period that is a multiple of the excitation, subharmonic motion, the Poincaré section will consist of a set of points. The Poincaré section of a chaotic trajectory has a fractal-like structure with an uncountable number of points, see Fig. 8 . The Poincaré section is chosen to be at the phase $2 \pi$ times the excitation frequency.

In the bifurcation diagrams shown in Figs. 9 and 10, velocity values from the Poincaré sections and velocities at impact are plotted versus the excitation frequency. To obtain these diagrams, a set of initial conditions are chosen and the equation of motion is solved. The transient part of the motion is neglected and the characteristics of the motion during the following 100 periods of excitation are plotted, at the current frequency. The frequency is then increased by one step and the last state of the system at phase $2 \pi$, from the previous frequency response, is taken as initial conditions for the new frequency. The procedure is repeated for the following frequency. The frequency is varied from 2 to $6 \mathrm{~Hz}$, with an increment of $0.01 \mathrm{~Hz}$.

These diagrams provide information about the expected motions, its relation to the frequency of excitation and its impact characteristics. In the impact velocity bifurcation diagrams, impacts with the upper stop are characterised by positive velocities, while impacts with the lower stop have negative velocities. 

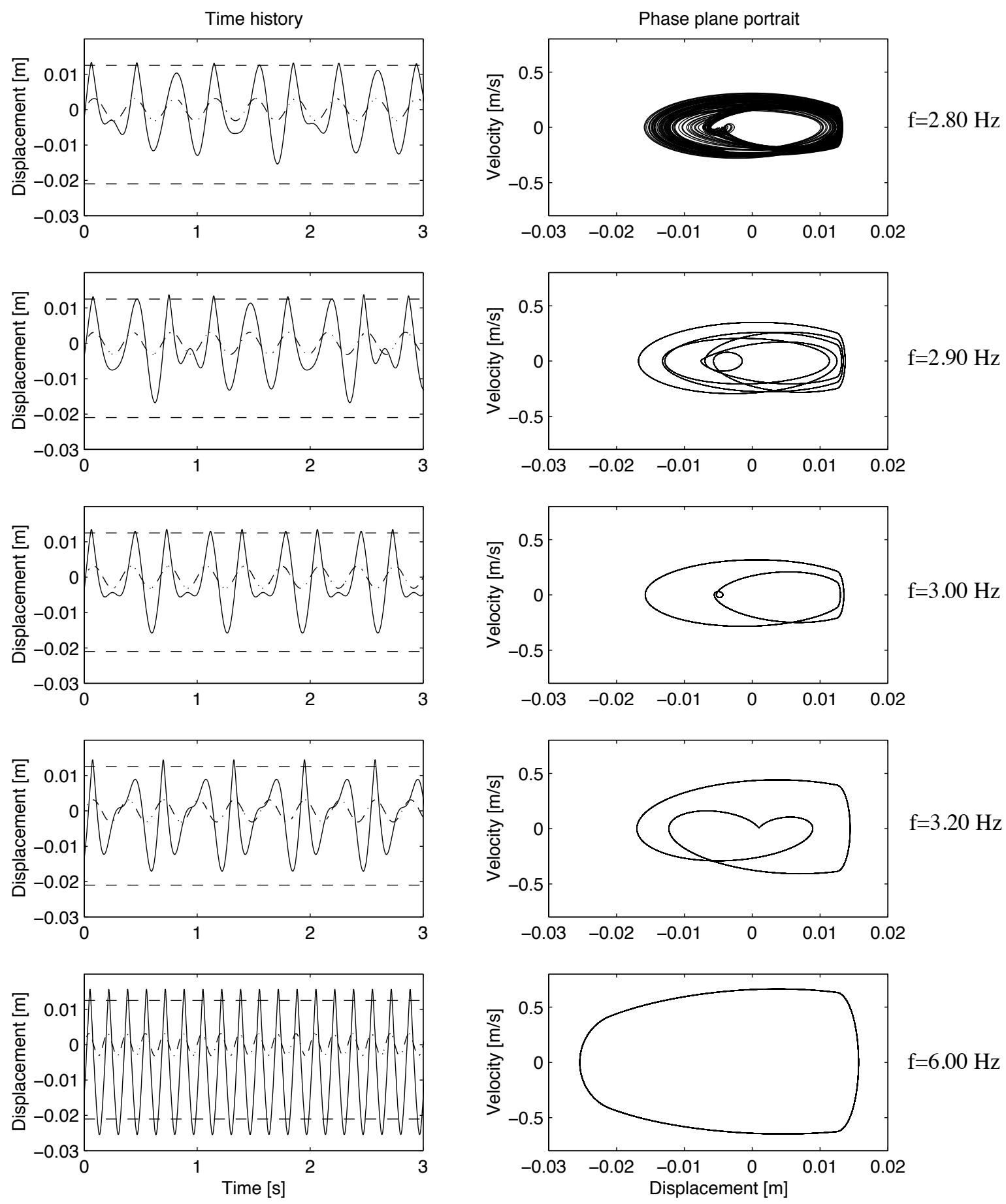

Figure 6. Samples of time histories and phase plane portraits of responses for Case 1. 

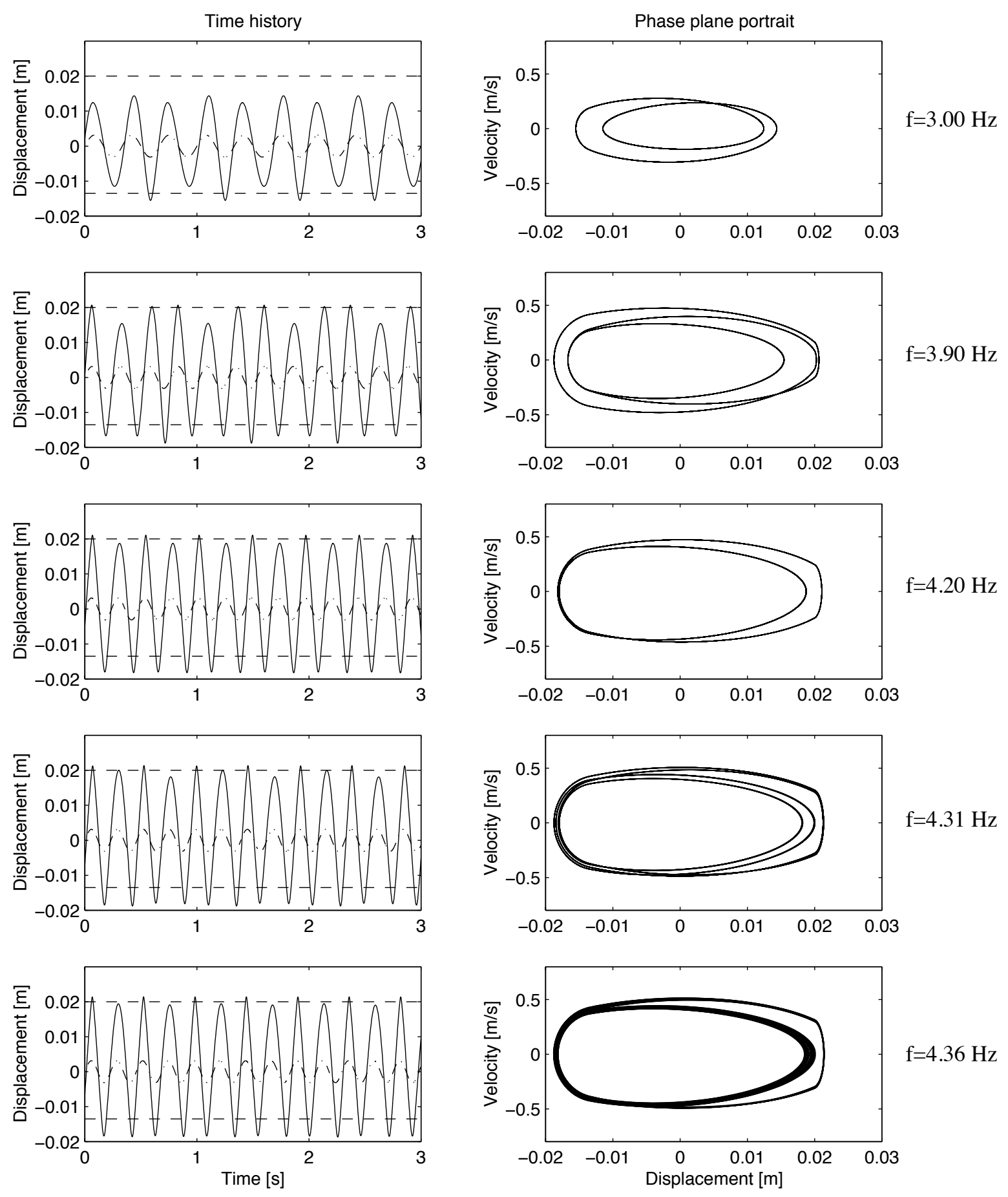

Figure 7. Samples of time histories and phase plane portraits of responses for Case 2. 


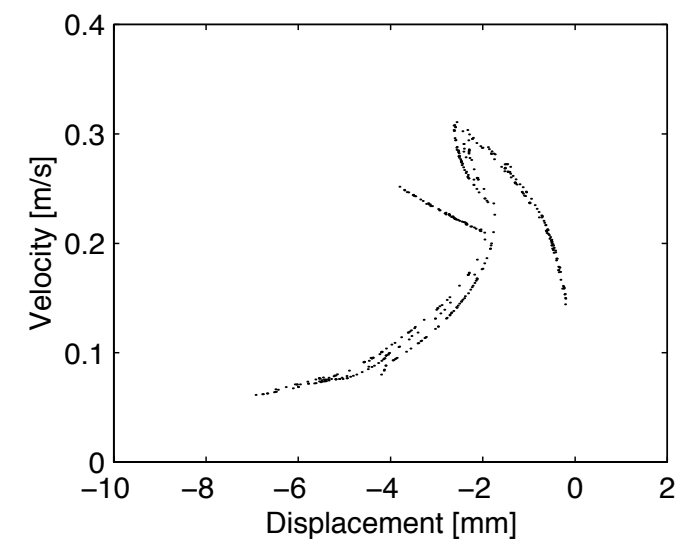

Figure 8. Poincaré diagram for Case 1 and an excitation frequency of $2.80 \mathrm{~Hz}$.
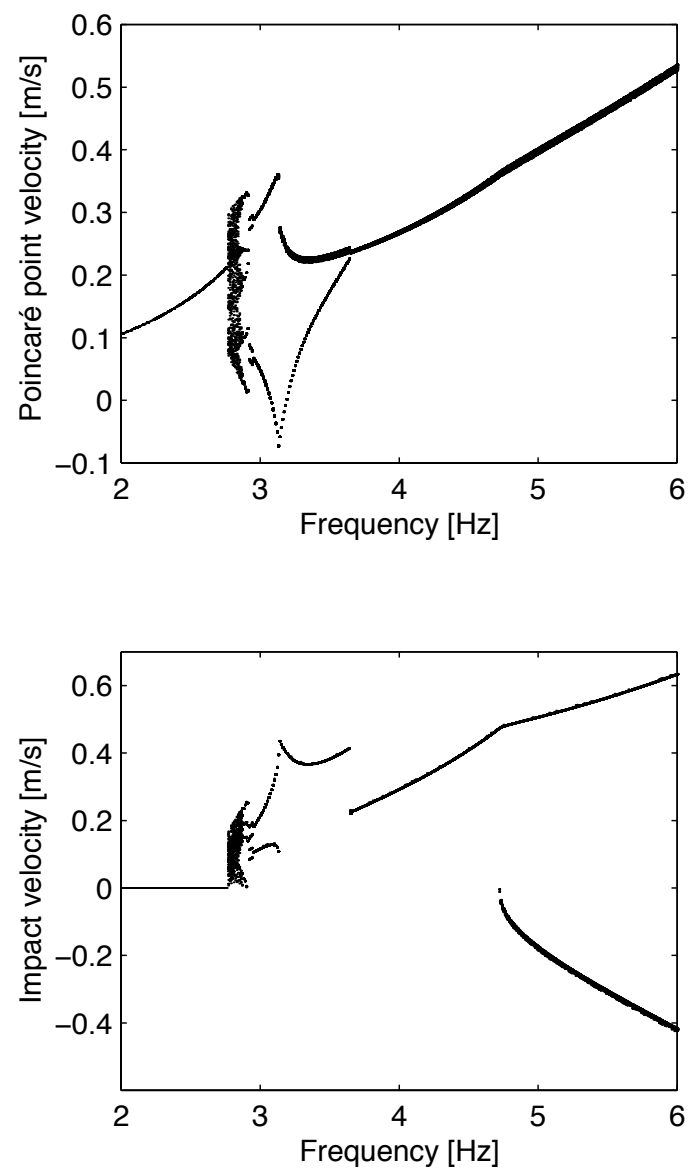

Figure 9. Bifurcation diagrams for Case 1.
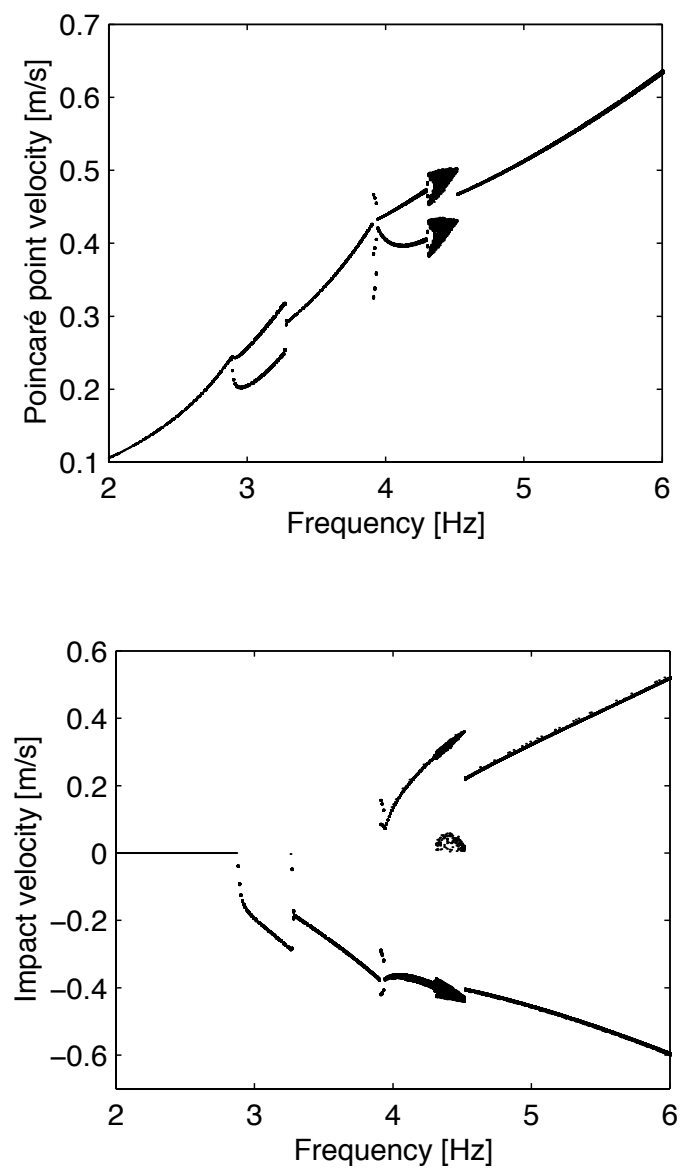

Figure 10. Bifurcation diagrams for Case 2.

\section{DISCUSSION AND CONCLUSIONS}

A nonlinear model of a pantograph suspension subsystem has been derived according to physical parameter values of the head suspension of the Schunk WBL88/X2 pantograph. In this model the pantograph is considered to be symmetrically excited with a harmonic and a bias force, and the main pantograph motion is assumed to occur in the head assembly. In reality, the dynamic behaviour of the system also depends on other factors, such as the characteristics of the frame assembly and the catenary. Nevertheless, in order to understand the behaviour of the system undergoing a more general excitation, it is of importance to first understand the dynamic behaviour of the subsystems due to harmonic excitation.

Fig. 6 illustrates different types of behaviour for Case 1. Periodic response with the same frequency as the excitation, subharmonic responses with periods that are two and five times the period of the excitation, and also a region with chaotic response occurs. The characteristics of the behaviour for different frequencies are illustrated in Fig. 9. From 2 to $2.76 \mathrm{~Hz}$ the response is periodic with the same period as the 
excitation. When the excitation frequency is increased to 2.77 $\mathrm{Hz}$ the mass starts to impact the upper stop. The response is irregular and the Poincaré sections show fractal-like structures implying chaotic behaviour, see Fig 8. At $2.87 \mathrm{~Hz}$ the response becomes periodic again with a subharmonic motion of period ten. At $2.88 \mathrm{~Hz}$ the response is subharmonic with a period five motion and this behaviour continues up to $2.92 \mathrm{~Hz}$ where a subharmonic motion of period four occurs. The periodicity decreases to a period two motion at $2.95 \mathrm{~Hz}$ and ends up with a response that is periodic with the same period as the excitation from 3.65 to $6 \mathrm{~Hz}$. For frequencies higher than $4.7 \mathrm{~Hz}$ the mass starts to impact both stops.

Fig. 7 illustrates different types of behaviour for Case 2 . Subharmonic responses with periods that are two, three and four times the period of the excitation, and finally an irregular behaviour occur. In Fig. 9 the characteristics of the behaviour for different frequencies are illustrated. From 2 to $2.87 \mathrm{~Hz}$ the response is periodic with the same period as the excitation. The mass starts to impact the lower stop when the excitation frequency is increased to $2.88 \mathrm{~Hz}$. The response is subharmonic with a period two motion up to $3.29 \mathrm{~Hz}$ where the response becomes periodic with the same period as the excitation again. The response continues to be a period one motion until $3.90 \mathrm{~Hz}$, where it becomes subharmonic with a period of three. The mass is now impacting both stops. At $3.94 \mathrm{~Hz}$ the response becomes subharmonic of period two. At $4.30 \mathrm{~Hz}$ the response is a period four motion. Between 4.34 and $4.49 \mathrm{~Hz}$ the response is irregular. At $4.50 \mathrm{~Hz}$ the response is subharmonic again with a period of two. Finally, the response is of period one from 4.51 to $6 \mathrm{~Hz}$.

The chaotic attractor shown in Fig. 8 has characteristics typical for impacting systems, i.e. the apparent disconnectedness of the attractor as well as the sharp corners. These are signs of the mass undergoing low velocity 'grazing' impacts (Stensson and Nordmark, 1994) which causes the system to become unstable. This is also visible in the bifurcation diagrams shown in Figs. 9 and 10. When low velocity impacts occur in the impact velocity diagrams, irregular motion is visible in the Poincaré point velocity diagrams.

To conclude, it is shown that nonlinear phenomena such as subharmonic motion and chaotic response can occur in a pantograph current collector suspension subsystem. These effects are therefore important to consider, when designing this type of product. It also put special demands on the analysis methodology used during the product development process since the dynamic behaviour can be very sensitive to changes in parameters and initial conditions.

\section{ACKNOWLEDGMENTS}

The financial support from the Swedish Research Council for Engineering Sciences and the Swedish Foundation for Strategic Research is gratefully acknowledged.

\section{REFERENCES}

Aidanpää, J-O, Chen H.H. and Gupta, R.B. 1994. Stability and Bifurcations of a Stationary State for an Impact Oscillator. CHAOS, 4 (2), 621-630.

ERRI. 1992. Pantograph/overhead line interaction. Question A 186, First draft, 2 July,1992.

Galeotti, G., Galanti, M., Magrini, S. and Toni, P. 1993. Servo Actuated Railway Pantograph for High-speed Running with Constant Contact Force. Proc. Instn. Mech. Engrs., 207, 37-49.

Larsson, T. and Drugge, L. 1998. Dynamic Behaviour of Pantographs due to Different Wear Situations. In Computers in Railways VI, Ed. B. Mellit et al., WIT Press, Southampton, 869-880.

Levy, S., Bain, J.A. and Leclerc, E.J. 1968. Railway Overhead Contact Systems, Catenary-Pantograph Dynamics for Power Collection at High Speeds. Journal of Engineering for Industry, 692-700.

Natsiavas, S. 1990. Stability and Bifurcation Analysis for Oscillators with Motion Limiting Constraints. Journal of Sound and Vibration, 141 (1), 97-102.

Natsiavas, S. and Gonzalez, H. 1992. Vibration of Harmonically Excited Oscillators with Asymmetric Constraints. Journal of Applied Mechanics, 59, 284-290.

Poetsch, G., Evans, J., Meisinger, R., Kortum, W., Baldauf, W., Weitl, A. and Wallaschek, J. 1997. Pantograph/Catenary Dynamics and Control, Vehicle System Dynamics, 28, 159-195.

Shaw, S.W. and Holmes, P.J. 1983. A Perodically Forced Piecewise Linear Oscillator. Journal of Sound and Vibration, 90, 129-155.

Stensson, A. and Nordmark, A. 1994. Experimental Investigation of some Consequences of Low Velocity Impacts in the Chaotic Dynamics of a Mechanical System. Philosophical Transactions of Royal Society of London A, 347, 439-448. 